\title{
Aplosporella ginkgonis (Aplosporellaceae, Botryosphaeriales), a new species isolated from Ginkgo biloba in China
}

\author{
Du $Z^{1}$, Fan $X^{1}$, Yang $Q^{1}$, Hyde $K^{2}$ and Tian $C M^{1 *}$ \\ ${ }^{1}$ The Key Laboratory for Silviculture and Conservation of Ministry of Education, Beijing Forestry University, Beijing \\ 100083, China \\ ${ }^{2}$ Center of Excellence in Fungal Research, Mae Fah Luang University, Chiang Rai 57100, Thailand
}

Du Z, Fan XL, Yang Q, Hyde KD, Tian CM 2017 - Aplosporella ginkgonis (Aplosporellaceae, Botryosphaeriales), a new species isolated from Ginkgo biloba in China. Mycosphere 8(2), 12461252, Doi 10.5943/mycosphere/8/2/8

\begin{abstract}
Aplosporella ginkgonis sp. nov., is described from symptomatic branches of Ginkgo biloba in China based on morphological and molecular analysis. It is characterized by multiloculate conidiomata, with one to four ostioles, and aseptate, brown, ellipsoid to oblong conidia. Morphological and phylogenetic analyses of ITS, and tef1- $\alpha$ sequence data, support the position of the new species in Aplosporella, which forms a monophyletic lineage with strong support (MP/BI = 100/1). Thus, a new species is introduced in this paper to accommodate this taxon.
\end{abstract}

Key words - Ascomycetous fungi - Canker disease - Dothideomycetes - Systematics - Taxonomy

\section{Introduction}

Aplosporella (Aplosporellaceae) was introduced by Spegazzini (1880) and has frequently been incorrectly synonymized, especially under Haplosporella (Tilak \& Rao 1964, Petrak \& Sydow 1927, Tai 1979, Wei 1979). In addition, the introduction of most new species was based on host occurrence, whereas recent studies suggest that taxa in this genus are not host-specific (Fan et al. 2015, Ekanayaka et al. 2016, Dou et al. 2017). More than 337 epithets for Aplosporella are listed in Index Fungorum (April 2017) with an estimated 66 species in Kirk et al. (2008).

Synonymies and frequent name changes in botryosphaerialean taxa, including Aplosporella, have caused much confusion for plant pathologists and mycologists (Dissanayake et al. 2016). Thus a systematic revision of Aplosporella is needed, which takes into account host, morphology and phylogenetic analysis. Revisiting all old epithets in Aplosporella would be ideal but is beyond most expectations (Dayarathne et al. 2016). Aplosporella is circumscribed by multiloculate conidiomata with a single ostiole, and brown, aseptate conidia. Slippers et al. (2013) recognized the genus should be separate from Botryosphaeriaceae as a distinct family, which they named Aplosporellaceae, and confirmed that Aplosporella is well-resolved with species described from living culture. However, Liu et al. (2016), using evolutionary evidence, questioned whether this family was introduced unnecessarily and should be regarded as a genus. Slippers et al. (2013) found a consistent clustering between Aplosporella and another similar genus Bagnisiella Speg. and considered that Bagnisiella represents the sexual morph and should be reduced to synonymy with Aplosporella (Schoch et al. 2009, Slippers et al. 2013). Ekanayaka et al. (2016) introduced a new species named Aplosporella thailandica from Thailand with similarities to Bagnisiella. Fresh 
collections of the latter genus are needed.

There are few reports of Aplosporella species from China, except for Haplosporella (currently Aposporella) species identified by host association and listed in Sylloge Fungorum Sinicorum and Identification of Fungus Handbook (Tai 1979, Wei 1979). Fan et al. (2015) isolated A. javeedii from five different hosts in China, which was previously regarded as an endophyte in South Africa (Jami et al. 2014). Subsequently, Dou et al. (2017) described A. macropycnidia from Cerasus yedoensis in China.

During studies on forest pathogenic fungi causing canker or dieback diseases in China, we unexpectedly isolated an Aplosporella species from Ginkgo biloba L. and Morus alba L. Following molecular and morphological study, we found this to be an undescribed species typical of Aplosporella. Therefore, in this paper we introduce Aplosporella ginkgonis sp. nov., with illustrations and compare it with other species in the genus.

\section{Materials \& Methods}

\section{Isolates}

Two specimens were collected from infected branches or twigs of Ginkgo biloba and Morus alba from Gansu in China. Two strains of Aplosporella were obtained following the methods of Chomnunti et al. (2014) (Table 1). The suspension of conidia was inoculated from the conidiomata on to the surface of $1.8 \%$ potato dextrose agar (PDA) in a Petri-dish, and incubated at $25{ }^{\circ} \mathrm{C}$ for up to $24 \mathrm{~h}$. Single germinating conidia were aseptically transferred to fresh culture plates. Living cultures are deposited and now maintained at the China Forestry Culture Collection Center (CFCC) and Beijing Forestry University (BJFU) under strain numbers CFCC 50465 and CFCC 50466. Specimens are deposited in the Museum of the Beijing Forestry University (BJFC) under collection numbers BJFC-S1313 and BJFC-S1314.

\section{DNA amplification, sequencing and phylogeny}

Fungal mycelium grown on PDA was scraped from the agar and used for genomic DNA extraction using a CTAB method (Doyle \& Doyle 1990). The ITS region was amplified using primers ITS1 and ITS4 (White et al. 1990). The tef- $1 \alpha$ region was amplified using EF1-688F and EF1-1251R primers (Alves et al. 2008). DNA sequencing was performed using an ABI PRISM® 3730XL DNA Analyzer with BigDye ${ }^{\circledR}$ Terminator Kit v.3.1 (Invitrogen) at the Shanghai Invitrogen Biological Technology Company Limited (Beijing, China). DNA sequences generated from this study were deposited in GenBank (Table 1), and a data matrix were deposited in TreeBASE (www.treebase.org) as accession S20694. The multiple sequence alignment of isolates selected from recent studies and GenBank were generated with MAFFT v.7 (Katoh \& Standley 2013).

Phylogenetic relationships were inferred by maximum parsimony (MP), and confirmed using MrBayes v.3.1.2 for Bayesian inference (BI) (Ronquist \& Huelsenbeck 2003). An MP search was performed using 1000 replicates of heuristic search with random addition of sequences and subsequent TBR branch swapping. Maxtrees were set to 5,000, branches of zero length were collapsed and all equally parsimonious trees were saved. Descriptive tree statistics (Tree Length [TL], Consistency Index [CI], Retention Index [RI] and Rescaled Consistency [RC] were calculated. Bayesian inference (BI) analysis employing a Markov Chain Monte Carlo (MCMC) method were performed with MrBayes (Rannala \& Yang 1996). A nucleotide substitution model was estimated by MrModeltest v.2.3 (Posada \& Crandall 1998). For the analysis, two MCMC chains were run simultaneously, starting from random trees for 1,000,000 generations, and trees were sampled every 100th generation for a total of 10,000 trees. The first $25 \%$ of trees were discarded as the burn-in phase of each analysis. Branches with significant Bayesian posterior probabilities (BPP) were estimated in the remaining 7,500 trees. Trees were visualized with FigTree v.1.4.0 (http://tree.bio.ed.ac.uk software/figtree) and the layouts were prepared with Adobe Illustrator CS v.6. 


\section{Morphology}

Characters of the fruiting bodies were observed on infected plant tissues and in culture. Macromorphological fungal structures were identified and studied with a Leica stereomicroscope (M205 FA), including size and arrangement of stromata, shape and size of ectostromatic disc and ostiole. Micromorphological observations with a Leica compound microscope (DM 2500) included the size and shape of conidiophores and conidia. More than 20 conidiomata were sectioned and 50 conidia were evaluated randomly for measurement. Cultural characteristics of strains incubated on PDA in the dark at $25{ }^{\circ} \mathrm{C}$ were recorded, including colony colour and conidiomata distribution. Taxonomic novelty was deposited in Faces of fungi numbers (FoF 02457) obtained as in Jayasiri et al. (2015).

Table 1 Strains of Aplosporella used in the molecular analyses in this study.

\begin{tabular}{|c|c|c|c|c|}
\hline \multirow[t]{2}{*}{ Species } & \multirow[t]{2}{*}{ Strain } & \multirow[t]{2}{*}{ Host } & \multicolumn{2}{|c|}{ GenBank Assession Number } \\
\hline & & & ITS & tef1- $\alpha$ \\
\hline Aplosporella africana & CBS 121777 & Acacia mellifera & EU101315 & EU101360 \\
\hline Aplosporella africana & CBS 121779 & Acacia mellifera & EU101317 & EU101362 \\
\hline Aplosporella africana ${ }^{\mathrm{T}}$ & CMW 25424 & N/A & KF766196 & EU101360 \\
\hline Aplosporella artocarpi ${ }^{\mathrm{T}}$ & СРC 22791 & Artocarpus heterophyllus & KM006450 & KM006481 \\
\hline Aplosporella hesperidica & CBS 208.37 & N/A & JX681069 & N/A \\
\hline Aplosporella javeedii & CFCC 50052 & Gleditsia sinensis & KP208838 & KP208844 \\
\hline Aplosporella javeedii & CFCC 50053 & Sophora japonica & KP208839 & KP208845 \\
\hline Aplosporella javeedii & CFCC 50054 & Juniperus chinensis & KP208840 & KP208846 \\
\hline Aplosporella javeedii $^{\mathrm{T}}$ & CFCC 89657 & Albizia julibrissin & KM030579 & KM030593 \\
\hline Aplosporella ginkgonis & CFCC 89660 & Morus alba & KR045623 & KP310847 \\
\hline Aplosporella ginkgonis ${ }^{\mathrm{T}}$ & CFCC 89661 & Ginkgo biloba & KM030583 & KM030597 \\
\hline Aplosporella macropycnidia ${ }^{\mathrm{T}}$ & CGMCC3.17725 & Cerasus yedoensis & KT343648 & KX011176 \\
\hline Aplosporella macropycnidia & CGMCC3.17726 & Cerasus yedoensis & KT343649 & KX011177 \\
\hline Aplosporella macropycnidia & CGMCC3.17727 & Cerasus yedoensis & KT343647 & KX011175 \\
\hline Aplosporella papillata ${ }^{\mathrm{T}}$ & CBS 121780 & Acacia tortillas & EU101328 & EU101373 \\
\hline Aplosporella papillata & CBS 121781 & Acacia erioloba & EU101329 & EU101374 \\
\hline Aplosporella papillata & CBS 121782 & Acacia erioloba & EU101330 & EU101375 \\
\hline Aplosporella prunicola ${ }^{\mathrm{T}}$ & CBS 121167 & $\begin{array}{l}\text { Prunus persica var. } \\
\text { nucipersica }\end{array}$ & KF766147 & N/A \\
\hline Aplosporella prunicola & STE-U 6326 & $\begin{array}{l}\text { Prunus persica var. } \\
\text { nucipersica }\end{array}$ & EF564375 & N/A \\
\hline Aplosporella prunicola & STE-U 6327 & $\begin{array}{l}\text { Prunus persica var. } \\
\text { nucipersica }\end{array}$ & EF564376 & N/A \\
\hline Aplosporella thailandica ${ }^{\mathrm{T}}$ & MFLU 16-0615 & N/A & KX423536 & KX423537 \\
\hline Aplosporella yalgorensis & MUCC 511 & Eucalyptus gomphocephala & EF591926 & EF591977 \\
\hline Aplosporella yalgorensis ${ }^{\mathrm{T}}$ & MUCC 512 & $\begin{array}{l}\text { Mimetes cucullata } \\
\text { Protea } \mathrm{sp}\end{array}$ & EF591927 & EF591978 \\
\hline Saccharata proteae ${ }^{\mathrm{T}}$ & CBS 115206 & N/A & KC343004 & KC 343730 \\
\hline
\end{tabular}

The new strains from the current study are in bold. Ex-type taxa are marked with a $\mathrm{T}$.

\section{Results}

\section{Phylogeny}

The combined ITS and tef1- $\alpha$ dataset from 23 ingroup strains (sequences of two strains 
from this study and sequences of 21 strains available in GenBank mostly from Mejía et al. 2011) clustered in 18 clades representing species of Aplosporella (Table 1). The alignment including gaps comprised 2864 characters of which 840 characters were constant, 467 variable characters were parsimony-uninformative, and 290 were parsimony informative. The heuristic search generated four equally parsimonious trees of 461 steps and $\mathrm{CI}=0.911, \mathrm{RI}=0.839, \mathrm{RC}=0.764$ as shown in Fig. 1. Strains CFCC 89961 and CFCC 89960, sequenced in this study, formed a well-supported clade $(\mathrm{MP} / \mathrm{BI}=100 / 1)$ representing a new phylogenetic species.

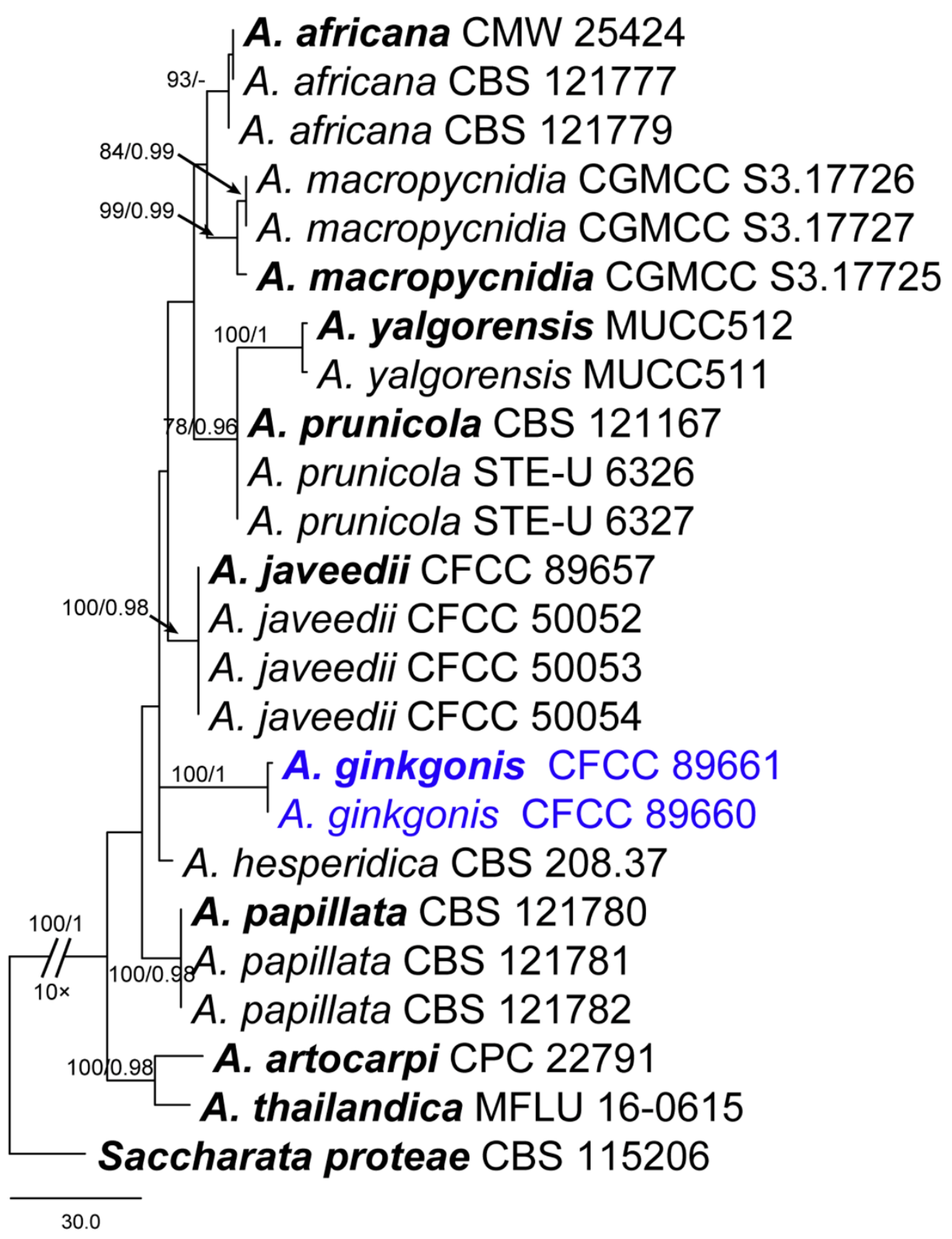

Figure 1 - Phylogram of Aplosporella based on analysis of combined ITS and tef1- $\alpha$ genes. MP bootstrap support values above $75 \%$ and BI posterior probabilities support above 0.95 from BI are shown above the branches. Scale bar $=30$ nucleotide substitutions. Ex-type strains are in bold. Strains in current study are in blue.

Taxonomy

Aplosporella ginkgonis C.M. Tian, Z. Du \& K.D. Hyde, sp. nov. Index Fungorum number: IF552938; Facesoffungi number: FoF 02457

Fig. 2 
Etymology - Named after the host genus, Ginkgo biloba.

Holotype - BJFC-S939.

Host/Distribution - from branches of Ginkgo biloba in China.

Diagnoses - The asexual morph of Aplosporella ginkgonis is similar to Aplosporella macropycnidia Zh. P. Dou \& Y. Zhang, which is from Cerasus yedoensis in China (Dou et al. 2017), but differs in its smaller pycnidia $(59-63 \times 77-81 \mu \mathrm{m}$ vs. $45-180 \times 38-138 \mu \mathrm{m})$.
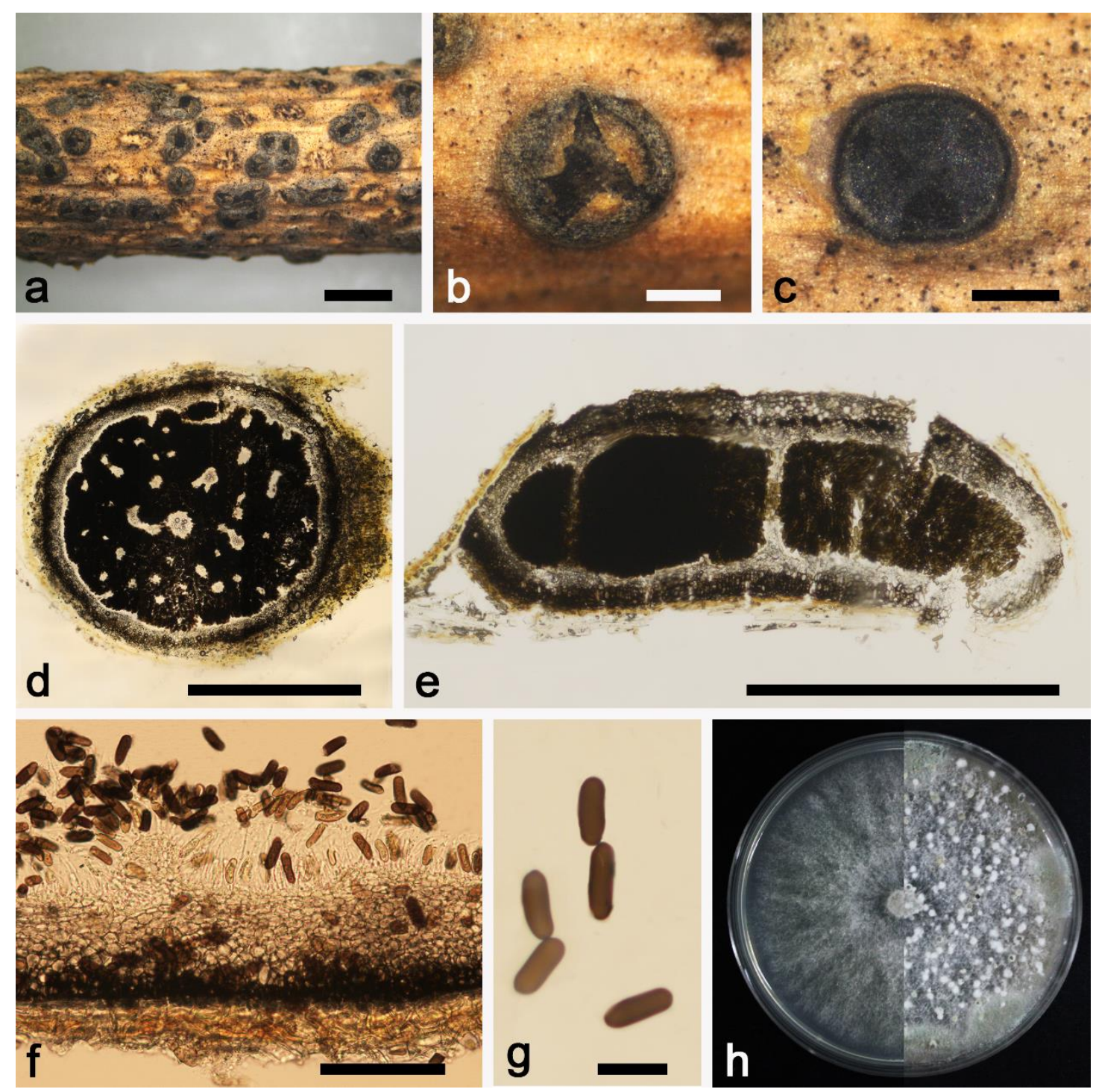

Figure 2 - Morphology of Aplosporella ginkgonis. a: Habit of conidiomata on twig. b: Multiostioles on the disc of conidiomata c, d: Transverse sections through conidiomata. e: Longitudinal sections through conidiomata. f: Conidiogenous cells and conidia. g: Conidia. h: Colonies on PDA at 3 days (left) and 30 days (right). Scale bars: $\mathrm{a}=2 \mathrm{~mm} ; \mathrm{b}-\mathrm{e}=0.5 \mathrm{~mm} ; \mathrm{f}=100 \mu \mathrm{m} ; \mathrm{g}=20 \mu \mathrm{m}$.

Sexual morph: Undetermined. Asexual morph: Conidiomatal stromata immersed in bark, erumpent from surface of the bark, separate, discoid, dark-brown to black, multiloculate, up to 850 $\mu \mathrm{m}$ diam., wall consisting of 8-12 layers, outer layers thick composed of dark-brown textura angularis, becoming hyaline and thin-walled towards the inner region. Disc brown to black, circular to ovoid, $(0.28-) 0.31-0.40(-0.44) \mathrm{mm}$ (average $=0.36 \mathrm{~mm}, \mathrm{n}=20$ ), with one to four ostioles per disc. Ostioles multiple, conspicuous, at the same level as the disc surface, covered below disc by lighter entostroma sometimes, $(22.5-) 23.9-30.7(-32.6) \mu \mathrm{m}$ (average $=28 \mu \mathrm{m}, \mathrm{n}=$ 20) in diam. Locules multiple, irregularly arranged, subdivided frequently by invaginations with common walls, $(0.59-) 0.63-0.77(-0.81) \mathrm{mm}$ (average $=0.71 \mathrm{~mm}, \mathrm{n}=20)$ in diam. Conidiogenous 
cells formed from the cells lining the inner walls of the locules, hyaline, smooth, inconspicuous. Conidia aseptate, ellipsoid to oblong, smooth, ends rounded, initial hyaline, becoming brown when mature, $(15.5-) 16-20.5(-21) \times(5.5-) 6.0-7.5(-8) \mu \mathrm{m}$ (average $=17.5 \times 6.5 \mu \mathrm{m}, \mathrm{n}=50)$.

Cultures - Cultures growth on PDA were initially white later producing a brown pigment on the reverse side after 7-10 days. Colonies were uniform with abundant white aerial mycelium.

Materials examined - CHINA. Gansu: Qingyang City, Qingyang Municipal Government, 35'42'23.21" N, 107³8'15.46" E, 1420 m asl., on stems of Ginkgo biloba, 14 Jul. 2013, collected by X.L. Fan (holotype, BJFC-S939, ex-type living culture, CFCC 89661); CHINA. Gansu, Qingyang City, Heshui County, 35 46'49.49"N, 107 59'31.48" E, 1277 m asl., on stems of Morus alba, collected by X.L. Fan, 13Jul. 2013, BJFC-S917, single conidium living culture CFCC 89660.

Notes - Morphologically, Aplosporella ginkgonis is characterized by its multilocular conidiomata with one to four ostioles, and aseptate, brown, ellipsoid to oblong conidia. Phylogenetically, two samples of $A$. ginkgonis formed a distinct lineage with high support (MP/BI $=100 / 1$ ) in the combined ITS and tef1- $\alpha$ analysis. Strains CFCC 89660 and 89661 had been misidentified as A. longipes based on host association; however, these strains differ by their larger conidia $(16-20.5 \times 6-7.5$ vs 12-20 × 5-6 $\mu \mathrm{m})$ and represent a unique species of Aplosporella from Ginkgo biloba (Saccardo 1899). Both morphology and the sequence data confirmed that the two samples represent a new species in Aplosporella using the guidelines for new species in Jeewon \& Hyde (2016).

\section{Acknowledgements}

This study is financed by National Natural Science Foundation of China (Project No.: 31670647) and the China Scholarship Council (CSC). We are grateful to Chungen Piao and Minwei Guo (China Forestry Culture Collection Center (CFCC), Chinese Academy of Forestry, Beijing) for support of strain preservation during this study.

\section{References}

Alves A, Crous PW, Correia A, Phillips AJL. 2008 - Morphological and molecular data reveal cryptic speciation in Lasiodiplodia theobromae. Fungal Diversity 28, 1-13.

Chomnunti P, Hongsanan S, Aguirre-Hudson B, Tian Q et al. 2014 - The sooty moulds. Fungal Diversity 66, 1-6.

Dissanayake AJ, Phillips AJL, Li XH, Hyde KD. 2016 - Botryosphaeriaceae: Current genera and species. Mycosphere 7, 1001-1073.

Dou ZP, Lu M, Wu JR, He W, Zhang Y. 2017 - A new species and interesting records of Aplosporella from China. Sydowia 69 (in press).

Doyle JJ, Doyle JL. 1990 - Isolation of plant DNA from fresh tissue. Focus 12, 13-15.

Dayarathne MC, Boonmee S, Braun U, Crous PW et al. 2016 - Taxonomic utility of old names in current fungal classification and nomenclature: Conflicts, confusion \& clarifications. Mycosphere 7, 1622-1648.

Ekanayaka AH, Dissanayake AJ, Jayasiri SC, To-anun C et al. 2016 - Aplosporella thailandica; a novel species revealing the sexual-asexual connection in Aplosporellaceae (Botryosphaeriales). Mycosphere 7, 440-447.

Fan XL, Yang Q, Cao B, Liang YM, Tian CM. 2015 - New record of Aplosporella javeedii on fve hosts in China based on multi-gene analysis and morphology. Mycotaxon 130, 749-756.

Jami F, Slippers B, Wingfield MJ, Gryzenhout M. 2014 - Botryosphaeriaceae species overlap on four unrelated, native South African hosts. Fungal Biology 118, 168-179.

Jayasiri SC, Hyde KD, Ariyawansa HA et al. 2015 - The Faces of Fungi database: fungal names linked with morphology, phylogeny and human impacts. Fungal Diversity 74, 3-18.

Jeewon R, Hyde KD. 2016 - Establishing species boundaries and new taxa among fungi: recommendations to resolve taxonomic ambiguities. Mycosphere 7, 1669-1677.

Katoh K, Standley DM. 2013 - MAFFT multiple sequence alignment software version 7: 
improvements in performance and usability. Molecular Biology and Evolution 30, 772-780.

Kirk PM, Cannon PF, Minter DW, Stalpers JA. 2008 - Ainsworth and Bisby's dictionary of the fungi, 10th edn. CAB International, Wallingford.

Liu NG, Ariyawansa HA, Hyde KD, Maharachchikumbura SSN et al. 2016 - Perspectives into the value of genera, families and orders in classification. Mycosphere 7, 1649-1668.

Mejía LC, Rossman AY, Castlebury LA, White JF. 2011 - New species, phylogeny, hostassociations and geographic distribution of genus Cryptosporella (Gnomoniaceae, Diaporthales). Mycologia 103, 379-399.

Petrak F, Sydow H. 1927 - Die Gattungen der Pyrenomyceten, Sphaeropsideen und Melanconieen. Beihefte Repertorium Specierum Novorum Regni Vegetabilis 42, 1-551.

Posada D, Crandall KA. 1998 - Modeltest: Testing the model of DNA substitution. Bioinformatics $14,817-818$.

Rannala B, Yang Z. 1996 - Probability distribution of molecular evolutionary trees: a new method of phylogenetic inference. Journal of Molecular Evolution 43, 304-311.

Ronquist F, Huelsenbeck JP. 2003 - MrBayes 3: Bayesian phylogenetic inference under mixed models. Bioinformatics 19, 1572-1574.

Saccardo PA. 1899 - Sylloge Fungorum vol 14. Typis Seminarii, Italy.

Schoch CL, Crous PW, Groenewald JZ et al. 2009 - A class-wide phylogenetic assessment of Dothideomycetes. Studies in Mycology 64, 1-15.

Slippers B, Boissin E, Phillips AJL, Groenewald JZ et al. 2013 - Phylogenetic lineages in the Botryosphaeriales: a systematic and evolutionary framework. Studies in Mycology 76, 3149.

Spegazzini C. 1880 - Fungi argentini. Pugillus tertius (Continuacion). Anales de la Sociedad Científca Argentina 10, 145-168.

Tai FL. 1979 - Sylloge Fungorum Sinicorum. Science Press, Beijing, China.

Tilak ST, Rao R. 1964 - The genus Haplosporella in India. Mycopathologia 24, 362-368.

Wei JC. 1979 - Identification of fungus handbook. Shanghai, China.

White TJ, Bruns T, Lee S, Taylor J. 1990 - Amplification and direct sequencing of fungal ribosomal RNA genes for phylogenetics. PCR protocols: a guide to methods and applications 18, 315-322. 